\title{
Affections dermatologiques de la muqueuse genitale masculine et de la verge
}

\author{
M. FAURE \\ Clinique dermatologique, Pavillon R, Hôpital E. Herriot, 69437 Lyon Cedex 3
}

\section{RESUME}

Les lésions de la verge, du gland et du sillon ont des causes très variées. Il peut s'agir de lésions de causes externes (eczémas, dermites d'irritation), de lésions infectieuses, de localisations génitales de dermatoses inflammatoires et même de processus tumoraux. Les lésions inflammatoires sont dominées par les balanites inflammatoires et par le lichen scléreux qui peuvent faire le lit du carcinome invasif. L'examen des autres muqueuses, l'examen du reste du tégument sont toujours utiles au diagnostic. Parfois le coup d'oeil dermatologique n'est pas suffisant. Il faut avoir recours à la biopsie d'une plaque érythémateuse, d'une érosion, d'une uilcération pour établir le diagnostic.

Mots clefs : balanite, carcinome, dermatologie, lichen, pénis.

\section{INTRODUCTION}

De nombreuses affections peuvent toucher les organes génitaux externes de l'homme, qu'il s'agisse de processus infectieux, de processus inflammatoires, les plus nombreux en fait, ou de processus tumoraux. Il peut s'agir d'une atteinte du gland, du sillon ou du sillon et du prépuce (balanite ou balano-posthite). Les mêmes affections peuvent se localiser aussi sur la verge, en particulier les localisations génitales des dermatoses inflammatoires.

La verge cependant peut être le siège de processus particuliers : la lymphangite sclérosante, après effort sexuel, peut prendre l'aspect d'un oedème préputial circonférentiel ou d'un cordon ferme depuis le sillon jusqu'à la base du pénis ; les kystes épidermiques ou dysembryoplasiques peuvent augmenter de volume, devenir inflammatoires, surtout après 20 ans, et sont de diagnostic aisé ; la maladie de La Peyronie enfin ou induration plastique des corps caverneux se voit surtout après 50 ans [5].

Le plus souvent cependant, l'aspect clinique est celui de plaques maculeuses ou papuleuses érythémateuses, d'érosions, ou même d'ulcérations qui n'ont pas de caractère séméiologique particulier permettant un diagnostic étiologique. L'aspect est " non spécifique ".Il s'agit d'une balanite et la séméiologie est identique que l'étiologie en soit infectieuse ou inflammatoire, avec un risque devant des lésions chroniques ou anciennes d'une évolution dysplasique et carcinomateuse. L'avis dermatologique est essentiel. C'est également l'interrogatoire qui oriente et précise les circonstances de survenue, les antécédents, le mode évolutif, aigu, à rechutes. Le diagnostic s'aide de la notion d'atteintes éventuelles d'autres muqueuses, de la constatation de lésions cutanées qui peuvent être évocatrices, de l'examen microscopique et éventuellement (balanites érosives) des données des examens immunopathologiques. 


\section{Dermatoses infectieuses}

Les causes les plus fréquentes sont les candidoses, l'herpès et les condylomes viraux.

\section{Candidose}

La balanite candidosique réalise une atteinte érythémateuse recouverte d'enduits blanchâtres (1). Elle est favorisée par l'obésité, le diabète, la macération, un défaut d'hygiène, un prépuce long. Parfois elle apparaît après une prise d'antibiotiques. Elle est souvent récidivante. Ce n'est que très exceptionnellement qu'elle doit être considérée comme à risque de transmission sexuelle. Les candidas albicans sont identifiés après mise en culture sur milieu de Sabouraud. Le traitement fait appel aux topiques azolés (laits, crèmes). Le recours à des antifongiques par voie générale n'est pas justifié, même en cas de candidose récidivante. En effet, ils ne traitent pas mieux, ni plus vite, ils n'empêchent aucunement les récidives, ils exposent à des risques non négligeables d'intolérance hépatique parfois sévère. En cas de candidose génitale récidivante, il est parfaitement inutile de traiter un éventuel foyer digestif, donc de le chercher, ou un foyer génital chez le, la partenaire. Le "terrain" peut être corrigé s'il existe, mais l'existence d'une balanite à répétition doit faire envisager le recours à une circoncision.

\section{Herpès génital}

Le diagnostic est facile soit devant une primoinfection herpétique, soit devant un herpès récidivant cyclique quand les signes cliniques sont évocateurs. L'herpès de primo-infection réalise un tableau de balanite érosive, avec un semis de lésions vésiculo-pustuleuses, un oedème préputial, une ou plusieurs adénopathies inguinales satellites, avec douleur et fièvre. L'herpès récidivant est évocateur devant la notion de lésions vésiculeuses et d'érosions du gland, du sillon, du pénis, précédées et accompagnées de sensations de cuisson, évoluant par poussées. Le diagnostic est confirmé par le frottis cellulaire (cytodiagnostic) qui montre des cellules épidermiques ballonisées (effet cytopathique). La sérologie n'a aucun intérêt pour le diagnostic de la nature herpétique d'une érosion génitale. La préven- tion des récidives, quand les poussées sont rapprochées donc invalidantes, repose sur la prise d'acyclovir (Zovirax ${ }^{\circledR} 800 \mathrm{mg}$ par jour) au long cours (pendant plusieurs années). Le traitement de la poussée fait appel à l'acyclovir $(200 \mathrm{mg}, 5$ fois par jour) ou la prise de valacyclovir (Zelitrex ${ }^{\circledR}, 1 \mathrm{~g}$ par jour en deux prises), avec alors diminution des signes cliniques, et raccourcissement de la durée de la poussée [8].

L'herpès génital peut être de diagnostic plus difficile. C'est alors le diagnostic d'une balanite érosive, d'un simple érythème. Il existe des formes très frustes, avec sensations de cuisson évoluant par poussées. Chez les sujets présentant un déficit immunitaire, (SIDA en particulier) le tableau est volontiers trompeur. C'est alors un aspect bâtard d'ulcération(s) génitale(s) chroniques, où manquent le caractère douloureux, les cuissons, l'évolution par poussées.

\section{Condylomes génitaux}

Les virus PVH (Virus des Papillomes Humains) sont responsables des verrues génitales, ou condylomes (végétations vénériennes). Le plus souvent il s'agit de lésions papillomateuses, papuleuses ou maculopapuleuses. Ces papillomes sont en nombre variable et peuvent siéger sur le sillon, le gland, au méat, sur le fourreau, le pénis et le pubis. Leur diagnostic est facile. Des lésions pigmentées du pénis peuvent cependant correspondre à des lésions tumorales d'autres natures : verrues séborrhéiques ou même naevus naevo-cellulaire. Les deux principaux diagnostics différentiels sont en fait représentés par les grains de Fordyce d'une part sur le fourreau et les papules perlées du gland. Ces hyperplasies glandulaires sont tout à fait physiologiques mais peuvent être un motif de consultation car d'inquiétude chez des hommes jeunes dans leur troisième décennie.

Parfois la contamination (sexuelle ou non sexuelle d'ailleurs) par les PVH donne des lésions différentes :

- rare condylome géant ou tumeur de Buschke-Löwenstein ;

- lésions de papulose bowenoïde : placards papuleux et pigmentés du fourreau et du 
pénis avec des caractères histologiques de dysplasie in situ comparables à ceux d'une maladie de Bowen ;

- condylomes dits plans, non décelables avant application d'acide acétique à $6 \%$, chez des partenaires de femmes atteintes de dysplasies du col utérin à PVH : macules acidophiles à la péniscopie, de taille et de nombre variables, du sillon, du gland, en zone donc muqueuse et semi-muqueuse [1].

La syphilis est devenue rare. Le chancre de la syphylis primaire est silencieux, indolore. Il s'agit d'une ulcération ou d'une simple érosion du sillon, du fourreau ou du pénis, accompagnée d'une adénopathie satellite. Se pose alors le diagnostic des autres causes d'érosions ou d'ulcérations génitales : traumatiques, caustiques, infectieuses (herpès, chancre mou), inflammatoires (aphtes en particulier) ou néoplasiques. Les lésions de syphilis secondaires sont en fait des macules ou des papules, indolores, asymptomatiques, entrant dans le cadre d'une roséole, ou bien de petites lésions cutanées du pénis et du périnée (condylomes tréponémateux). Faire le diagnostic de syphilis, c'est en fait y penser et demander les sérologies VDRL, TPHA et un FTA quantitatif. Le chancre primaire survient cependant en phase présérologique et une réponse VDRL-TPHA négative n'élimine pas ce diagnostic devant un complexe primaire. Une nouvelle sérologie doit alors être demandée. En cas de doute un traitement par pénicilline est entrepris [4].

La gale humaine réalise un tableau prurigineux génital et extragénital, à recrudescence nocturne. Les lésions génitales sont évocatrices quand existent des lésions papuleuses et papulonodulaires (nodules scabieux) du gland, du pénis, du scrotum [5].

\section{LÉSIONS INFLAMMATOIRES}

L'aspect le plus souvent est celui de lésions érythémateuses, mais parfois le tableau est celui d'une balanite ou balanoposthite érosive ou ulcéro-érosive. Ces lésions inflammatoires correspondent le plus souvent à des localisations génitales de dermatoses inflammatoires. L'interrogatoire, la recherche de localisations identiques ou comparables de la muqueuse buccale, celle de localisations cutanées à distance sont des étapes nécessaires au diagnostic. Parfois, seul l'examen anatomopathologique après biopsie cutanéo-muqueuse sous anesthésie locale permet le diagnostic.

\section{BALANITES DE CAUSES EXTERNES}

L'eczéma : il s'agit d'une balanite érythémateuse ou érosive, prurigineuse, de début brutal. Parfois le tableau est celui d'un oedème préputial. Cette réaction allergique de contact [5] peut survenir après application d'allergènes très divers : produits d'hygiène, produits de toilette, parfums, déodorants, autres produits de cosmétologie, préservatifs en latex, lubrifiants, médicaments topiques (antiseptiques, antibiotiques, crèmes diverses), etc...

Le traitement fait appel à des applications de crèmes cortisonées. Le diagnostic est confirmé par la positivité des tests cutanés (patch tests) au produit(s) suspecté(s).

Les dermites d'irritation ou dermites orthoergiques correspondent à des balanites le plus souvent érosives [5]. Il n'y a pas de caractère prurigineux. Il s'agit de sensations de brûlures, à la différence des dermites allergiques (eczémas de contact). Elles sont souvent liées à l'abus de produits d'hygiène, de savons, de produits de toilette, à des applications d'antiseptiques mais peuvent aussi être causées par des topiques médicamenteux (podophylline, Condyline ${ }^{\circledR}$ par exemple).

\section{LES TOXIDERMIES}

Lérythème pigmenté fixe : Les lésions surviennent brutalement sous forme d'une plaque érythémateuse et pigmentée sur le pénis, souvent associée à des plaques du reste du corps. L'évolution se fait vers l'atténuation en quelques semaines. La récidive survient lors d'une nouvelle prise du médicament responsable (9). L'érythème pigmenté fixe peut être déclenché par de nombreux médicaments: sulfamides, tétracyclines, amidopyrine, phénacétine, halogènes...

Les toxidermies bulleuses : Il s'agit d'une balanite érosive et croûteuse isolée, ou asso- 
ciée à des lésions érosives des autres muqueuses, à des lésions péri-orificielles (syndrome de Stevens-Johnson), parfois à un exanthème sur le reste du tégument avec des lésions ressemblant aux lésions en cocarde d'un érythème polymorphe. Le début est brutal. Ces toxidermies surviennent volontiers après prise d'anti-inflammatoire non stéroïdien ou de sulfamide. L'évolution se fait en quelques jours vers la décrustation et la cicatrisation. En l'absence de lésions cutanées associées, les lésions sont volontiers confondues avec un herpes génital. L'existence d'une atteinte bipolaire, buccale et génitale, doit attirer l'attention. Ce type d'atteintes érosives bipolaires, qu'il y ait ou non une atteinte cutanée associée, peut cependant compliquer l'évolution d'une poussée herpès récurrent buccal ou génital tout à fait banale. C'est l'anamnèse qui permet de trancher : absence d'antécédents herpétiques et existence d'une prise médicamenteuse dans les jours qui ont précédé $[3,9]$.

\section{LAPHTOSE}

Les aphtes génitaux peuvent siéger sur les muqueuses, mais également sur la peau du pénis ou du scrotum. Il s'agit d'ulcérations douloureuses, soit de petites ulcérations superficielles à fond " beurre frais ", prises à tort pour des lésions herpès, soit d'ulcérations franches, de grande taille, profondes parfois géantes, nécrotiques, simulant un chancre. Ces aphtes peuvent se rencontrer dans trois circonstances. Il peut s'agir d'une simple aphtose génitale récidivante, d'une aphtose bipolaire, avec atteinte des muqueuses génitales et buccales, ou encore d'une grande aphtose, avec poussées de lésions muqueuses, cutanées, oculaires (syndrome de Behcet), et manifestations viscérales, thromboemboliques en particulier [5].

\section{LE PSORIASIS}

Les lésions sur le gland et le sillon correspondent à des plaques maculeuses érythémateuses en cartes de géographie [5]. Sur le versant cutané peut exister le caractère plus évocateur de lésions papuleuses érythématosquameuses. Le diagnostic est facilité par l'existence de lésions en plaques papulo-squameuses cutanées sur les coudes, le tronc, les membres inférieurs, par un intertrigo inguinal et périnéal avec atteinte du pli interfessier. Sinon une biopsie est nécessaire pour le distinguer d'une autre cause d'érythroplasie génitale. L'évolution est en général chronique ou subchronique, avec des poussées spontanées et à chaque fois une évolution favorable sous dermocorticoïdes, qui n'évitent cependant pas les récidives.

\section{LE LICHEN}

Il peut prendre deux aspects [5].

le lichen plan cutané et muqueux : Il s'agit d'une éruption papuleuse ou micropapuleuse du gland ou du pénis, prurigineuse. Les lésions sont évocatrices quand existent des lésions papuleuses et prurigineuses dans des territoires cutanés d'élection : chevilles, poignets, tronc. Sinon l'examen microscopique est nécessaire pour le diagnostic montrant l'infiltrat lymphocytaire caractéristique du lichen plan. Deux diagnostics doivent en fait être évoqués devant l'existence de papules prurigineuses des organes génitaux masculins : le lichen plan et la gale humaine. L'évolution est bénigne, avec guérison spontanée ou sous dermocorticoïdes. L'étiologie est inconnue. Certains lichens sont liés au stress, à l'angoisse.

le lichen érosif : La nécrose épidermique prédomine sur la réaction lymphocytaire. Il s'agit alors d'une balanite érosive très douloureuse et d'évolution chronique, à la différence du lichen plan simple. Il n'existe pas de lésions cutanées. Il peut cependant exister une atteinte buccale, linguale, gingivale (forme bipolaire). Il s'agit alors du diagnostic d'une balanite érosive et les examens anatomopathologiques et immunopathologiques sont nécessaires au diagnostic, pour faire le diagnostic de lichen érosif et éliminer une autre cause d'érosion génitale, une localisation de dermatose bulleuse auto-immune en particulier. Une fois le diagnostic posé, la prise en charge est difficile. Les moyens thérapeutiques sont décevants : corticothérapie locale ou générale, antipaludéens de synthèse, immunosuppresseurs... Une surveillance biannuelle est nécessaire, 
car le risque est celui d'une dégénérescence carcinomateuse.

\section{LES DERMATOSES BULLEUSES AUTO-IMMUNES.}

Le diagnostic est facile quand existent des lésions cutanées qui ont déjà permis le diagnostic. Les érosions génitales peuvent être cependant isolées. Le tableau est alors celui d'une balanite érosive. Le plus souvent il s'agit d'une dermatose bulleuse liée à un décollement au niveau de la jonction dermoépidermique : pemphigoïde à IgG ou IgA, pemphigoïde muco-synéchiante ou cicatricielle [2]. Plus rarement il s'agit d'un pemphigus auto-immun avec décollement intra-épidermique par acantholyse.

La recherche de lésions érosives, bulleuses ou croûteuses cutanées doit être systématique, mais ces atteintes peuvent manquer. Le recherche systématique d'une atteinte d'autres muqueuses (bouche, oeil) est nécessaire. Le siège du décollement peut être affirmé par l'examen anatomopathologique. La caractérisation du type de dermatose bulleuse nécessite en fait la mise en évidence d'anticorps fixés par immunofluorescence (IF) directe après biopsie muqueuse ou cutanée et du siège des dépôts d'IgG ou IgA (sous épidermiques ou épidermiques intercellulaires) et d'anticorps dans le sang circulant par IF indirecte [7].

La gravité de ces affections rend indispensable un diagnostic précoce et justifie le recours à ces examens complémentaires devant toute balanite érosive suspecte de correspondre à une localisation génitale de dermatose auto-immune.

\section{LE LICHEN SCLÉREUX GÉNITAL MASCULIN}

Il prend l'aspect d'une balanite érythémateuse et prurigineuse d'installation insidieuse. Il peut aussi être révélé brutalement par un phimosis ou un paraphimosis. Il peut être diagnostiqué à un stade tardif avec rétrécissement et sclérose du prépuce, c'est le stade de kraurosis pénis. Le diagnostic est souvent en fait facile devant l'existence de plages leu- coplasiques, blanc nacré, associées à des zones érythroplasiques, vernissées, parfois indurées à la palpation. La biopsie confirme le diagnostic en montrant l'aspect hyalin du derme sous épidermique avec en profondeur un infiltrat lymphocytaire. Parfois il s'agit d'érosions ou d'ulcérations du gland ou du sillon, parfois de plages hémorragiques. La sclérose peut s'étendre à la peau génitale, avec un véritable anneau de striction (kraurosis). Le lichen scléreux peut faire le lit d'une dysplasie épithéliale (maladie de Bowen) pouvant évoluer vers la constitution d'un carcinome spino-cellulaire. C'est dire l'importance d'un diagnostic précoce, devant toute balanite chronique ou à rechute, et souligner la nécessité d'une circoncision, seule façon devant un lichen scléreux génital masculin même débutant d'éviter l'évolution vers le kraurosis et/ou la dégénérescence [5].

\section{LES AUTRES BALANITES INFLAMMATOIRES}

Certains hommes présentent après ou même avant 50 ans des poussées de lésions érythémateuses, cuisantes ou prurigineuses avec une atteinte diffuse ou en plaques du sillon, du gland, du prépuce. Il n'existe pas de signe clinique permettant d'évoquer un lichen scléreux (ni induration, ni aspect ivoiré, ni kraurosis). Les traitements antifongiques sont inefficaces, les dermocorticoïdes aussi. La biopsie montre une image d'infiltration plasmocytaire (Balanite de Zoon) ou lympho-plasmocytaire. L'évolution de ce type de balanite chronique se fait par alternances de poussées et de rémissions [5]. Elle peut cependant faire le lit d'une transformation dysplasique. La circoncision doit être proposée, seul moyen d'obtenir en fait une guérison.

\section{TUMEURS ET PROCESSUS DYSPLASIQUES}

Le mélanome malin est rare dans cette localisation [5]. Il doit être distingué en fait de naevus bénins, des taches noires de la pigmentation mélanique essentielle des muqueuses (maladie de Laugier) sur le gland, le sillon et la peau pénienne. D'autres lésions pigmentées de la peau génitale peu- 
vent en fait correspondre à des condylomes génitaux ou à de simples verrues séborrhéiques.

Des lésions pigmentées ou bleutées peuvent par ailleurs correspondre à des angiomes, mais aussi à de maladie de Kaposi lors de SIDA. La localisation génitale est plus rare que l'atteinte buccale. Les lésions sont soit des papules violacées, soit au stade initial de simples plaques maculeuses sans relief.

\section{CARCINOMES ET DYSPLASIES " PRÉ- CARCINOMATEUSES "}

Le carcinome spino-cellulaire survenant sur une muqueuse saine est rare. Il s'agit le plus souvent d'une lésion ulcéro-végétante, parfois d'une forme infiltrée qui complique un lichen scléreux génital ou une balanite chronique ou à rechutes. Toute lésion génitale érosive ou ulcérée chronique doit en fait être biopsiée pour contrôle anatomopathologique. En fait le carcinome spino-cellulaire invasif survient comme stade évolutif d'une dysplasie intraépithéliale (PIN: néoplasies intraépithéliales du pénis). L'érythroplasie de Queyrat est l'appellation dermatologique clinique classique, historique qui correspond au carcinome in situ ou maladie de Bowen muqueuse (PIN III). Il s'agit d'une lésion érythroplasique d'évolution chronique. La biopsie fait le diagnostic. Ces dysplasies surviennent le plus souvent après $5 \mathrm{O}$ ans chez le sujet non circoncis comme évolution d'une balanite chronique ou d'un lichen scléreux. Plus rarement, et alors surtout en cas de déficit immunitaire (transplantés d'organe en particulier), il s'agit de lésions survenant sur des lésions de papulose bowenoïde, ou sur une muqueuse génitale saine, mais liée, à la différence du carcinome classique, à une infestation de virus PVH. Le traitement est chirurgical ou curiethérapique [6].

\section{CONCLUSION}

Les lésions de la muqueuse génitale masculine, et de la peau génitale peuvent être de nature et de mécanismes très variés. On retrouve la richesse de la dermatologie elle même. Le diagnostic peut parfois être facile, de coup d'oeil, en cas d'oeil exercé. Il est souvent plus difficile. Il s'aide de l'examen des autres muqueuses et du reste du tégument, de l'examen anatomopathologique et même parfois d'examens d'immunopathologie. En dehors des affections cutanées et génitales infectieuses (herpès, candidoses, condylomes), il s'agit du diagnostic d'une balanite qui représente la question la plus fréquente en pratique médicale quotidienne. Le point le plus important est d'éviter l'évolution vers une dysplasie épithéliale. Le recours à une circoncision est souvent la seule attitude thérapeutique à proposer.

\section{REFERENCES}

1. ACHTEN G, ANDRÉ J, CLUMECK, N, DE MAUBEUGE J, GOENS J, PARENT D: Les grands syndromes des MST. Encycl. Med. Chir. (Paris-France), Dermatologie, 12670 B10, 7-1988, 18p

2. AHMED AR, KURGIS BS, ROGERS RS: Cicatricial Pemphigoid. J. Am. Acad. Dermatol., 1991, 24: $987-$ 1001

3. BASTUJI-GARIN S, CHOSIDOW O, REVUZ J, ROUJEAU JC: Erythème polymorphe. Editions Techniques. Encycl. Med. Chir. (Paris-France), Dermatologie, 12-450-B-10, 1993, 5p

4. CAUMES E, JANIER M: Syphilis. Editions Techniques. Encycl. Med. Chir. (Paris-France), Maladies infectieuses, 8-039-C-10, 1995, 14p

5. COLOMB D. Affections de la muqueuse génitale masculine et de la verge. In: Saurat JH, Grosshans E, Laugier E, Lachapelle JM eds. Dermatologie et Vénéréologie, Paris, Masson, 1990, 677-681

6. PARNEIX-SPAKE A, SARRET Y, THIVOLET J, FAURE M: Le traitement des carcinomes spiono-cellulaires de la verge par chirurgie et par curiethérapie, Ann. Dermatol. Venereol., 1993, 120: 409-415

7. STANLEY JR: Pemphigus et Pemphigoid as paradigms of organ-spécific autoantibody-mediated diseases. J. Clin. Invest., 1989, 83: 1443-1448

8. TYRING SK, DOUGLAS JM, COEY L, Spruance SL, Esmann J: A randomized, placebo-controlled comparison of oral valacyclovir and acyclovir in immunocompetent patients with recurrent genital herpes infections,. Arch. Dermatol., 1998, 134: 185-191

9. WOLKENSTEIN P, ROUJEAU JC: Toxidermies. Editions techniques. Encycl. Med. Chir. (Paris-France), Dermatologie, 12-930-A-10, 1995, 7p 


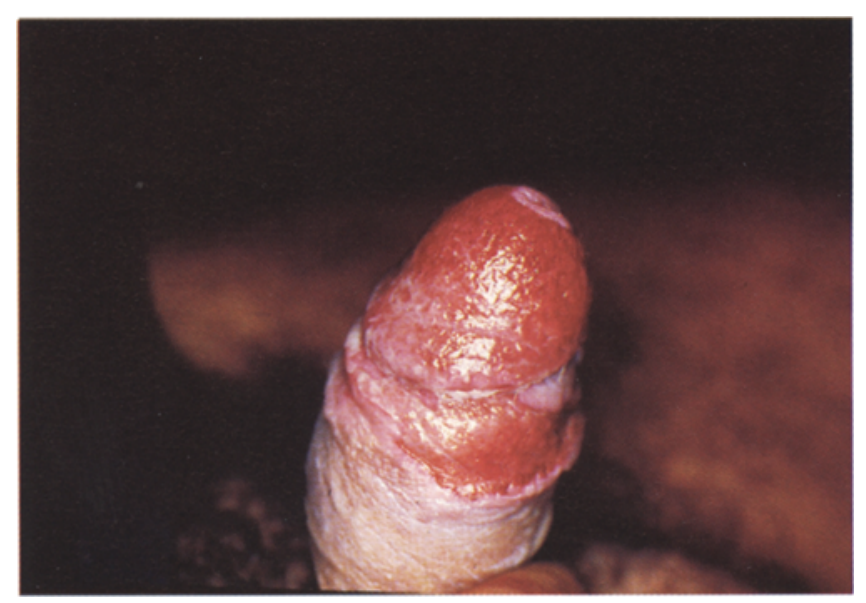

Figure 1: Balanite caustique

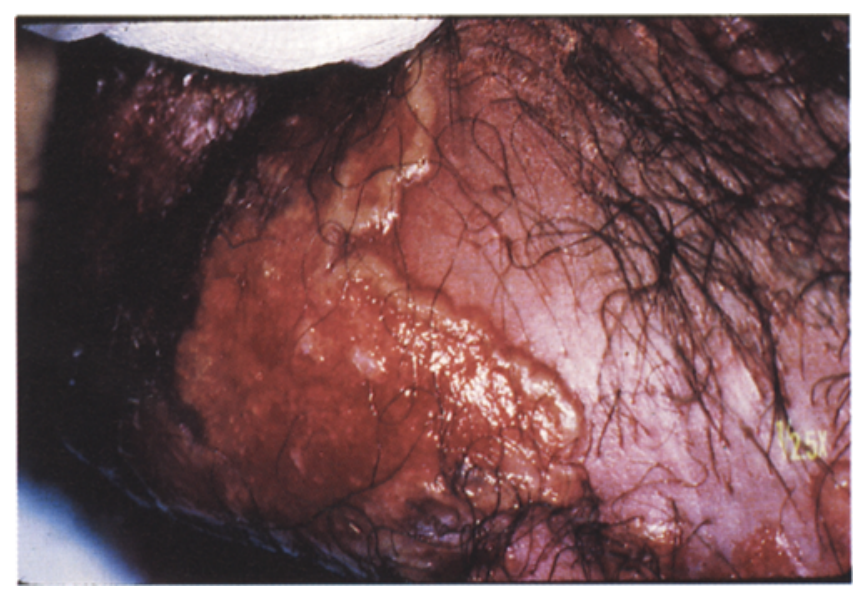

Figure 3: Ulcération herpétique

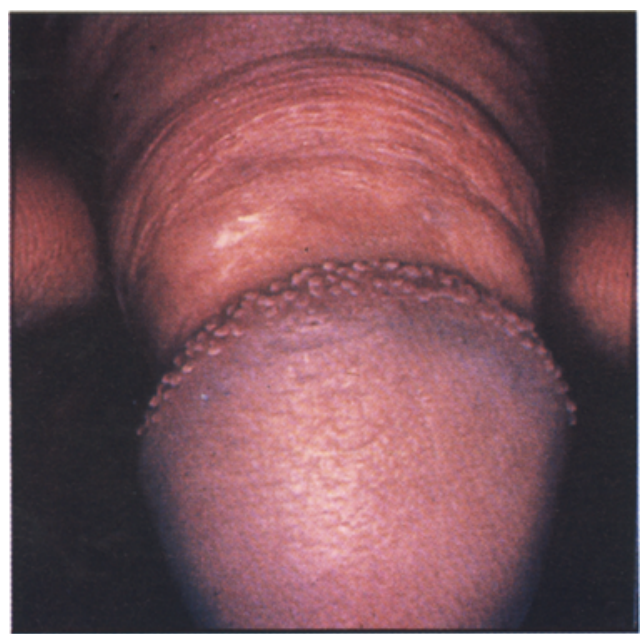

Figure 5: Ppules perlées physiologiques du gland

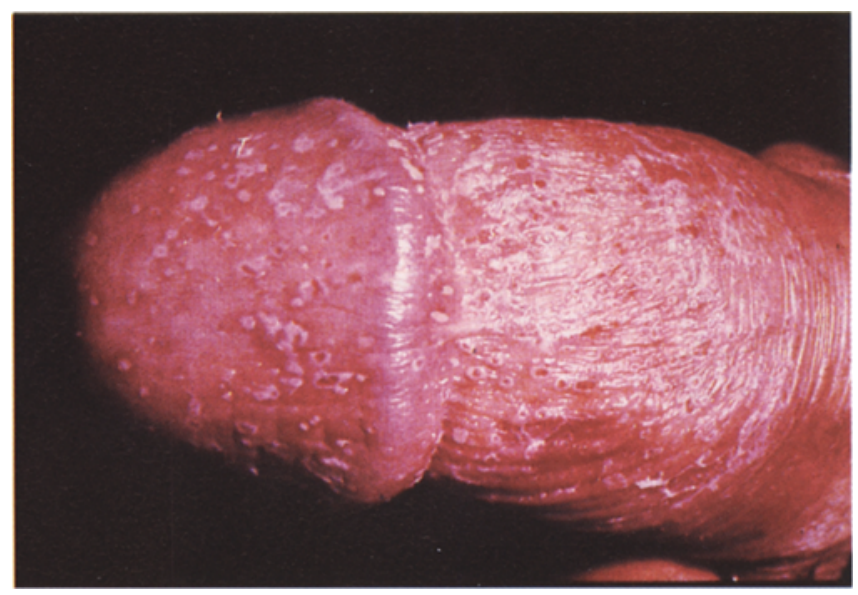

Figure 2: Balanite candidosique

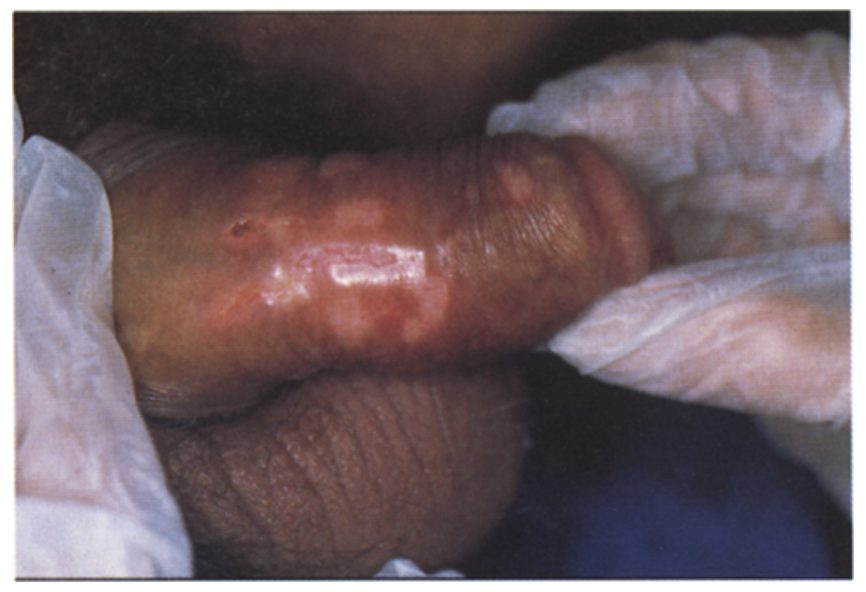

Figure 4: Lésions herpétiques chez un sidéen

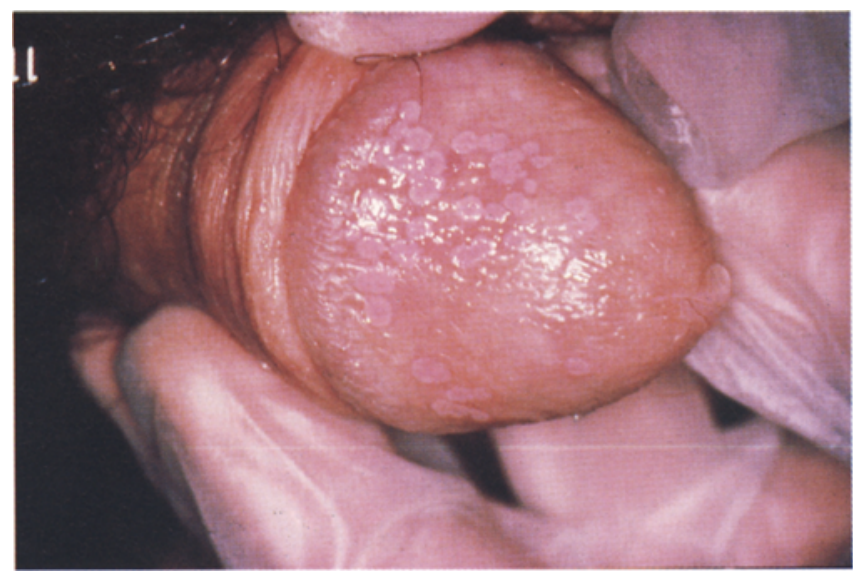

Figure 6: Condylomes "plans" après application d'acide acétique 


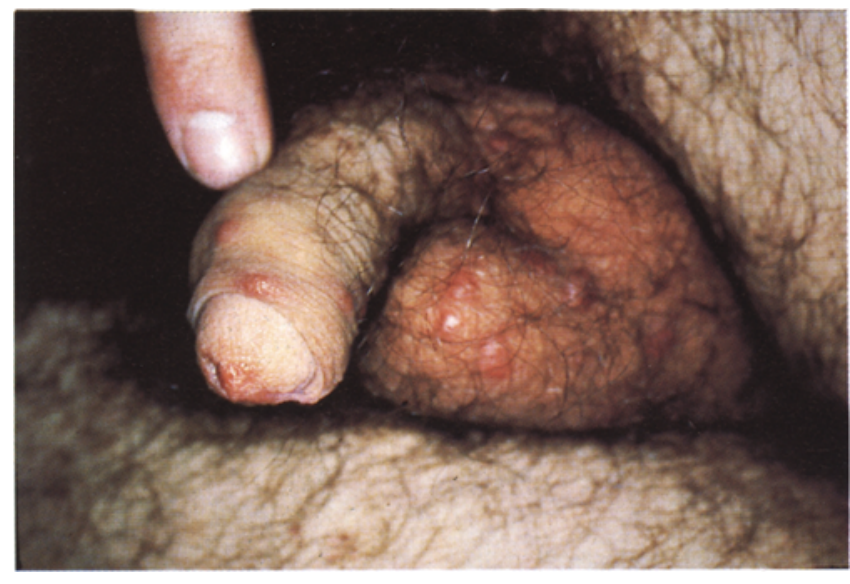

Figure 7: Nodules scabieux

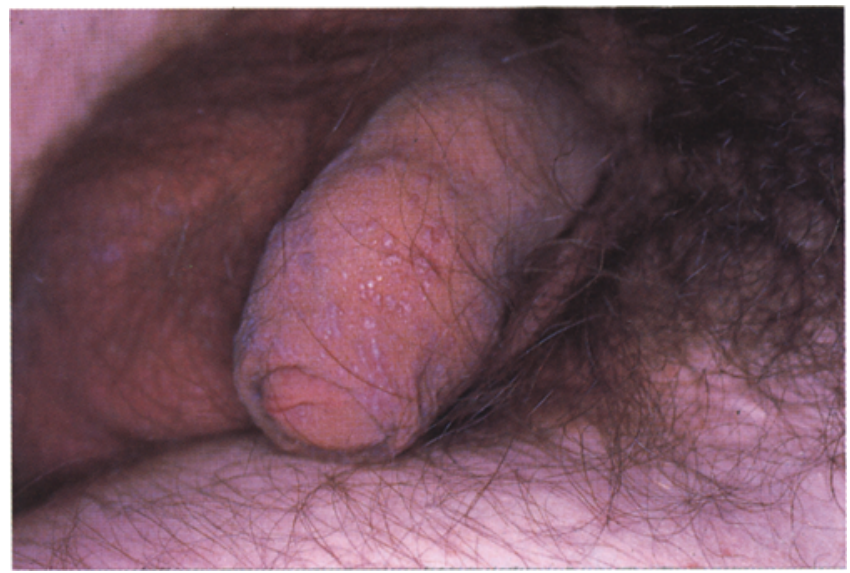

Figure 9: Lichen plan cutané génital

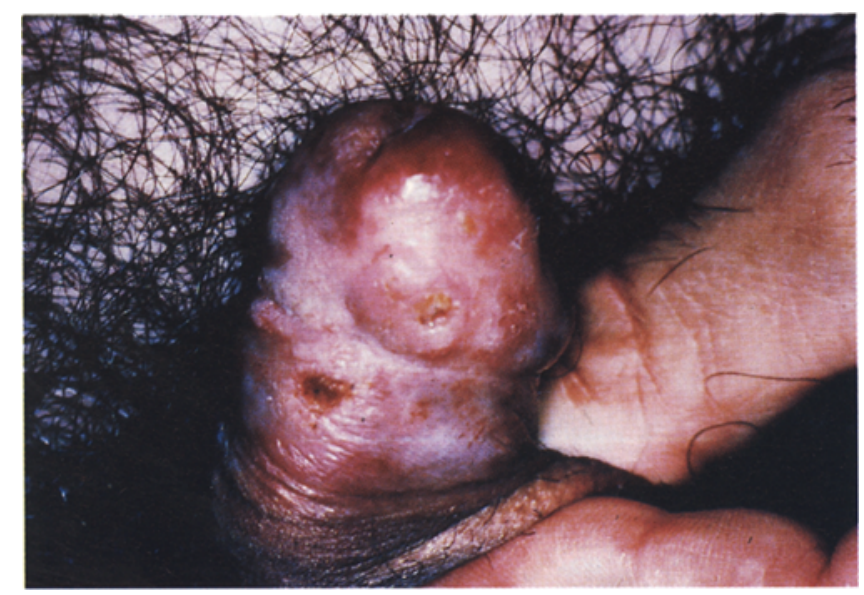

Figure 11: Carcinome sur lichen scléreux.

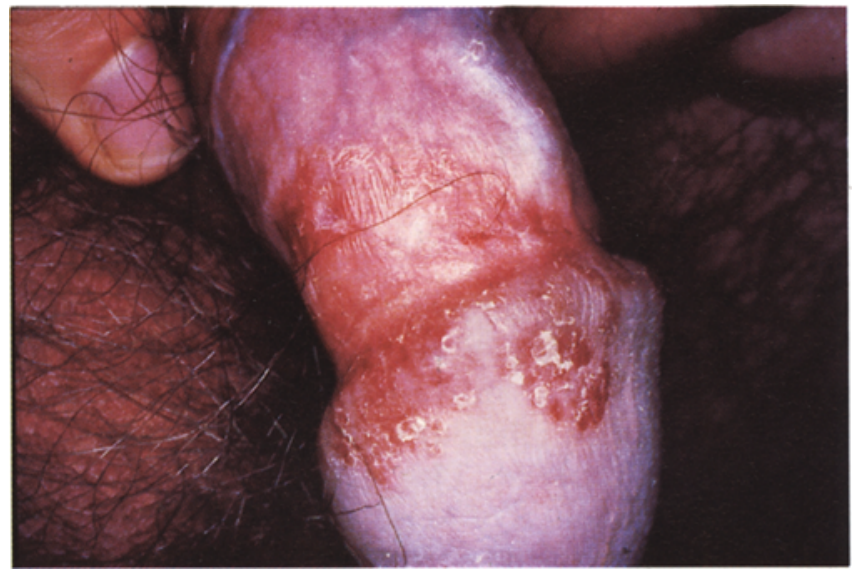

Figure 8: Psoriasis génital

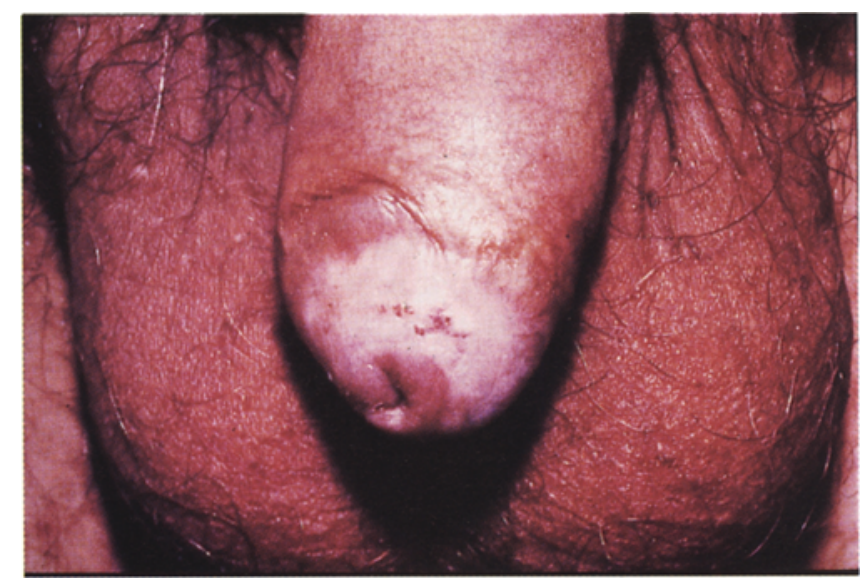

Figure 10: Lichen scléreux génital 


\begin{abstract}
Dermatologic disorders affecting male genital skin and mucous membranes

\section{FAURE}

A wide variety of infectious, inflammatory or dysplasic disorders may affect both male genital skin and mucous membranes. We review here the clinical patterns or the most common disorders. The diagnosis is based upon the existence of erythematous balanitis, erosions or ulcerations. Among infections, candidiosis, genital herpes, HPV papillomas are the most common. Most of inflammatory skin disorders may affect the male organ, especially psoriasis, lichen planus or erosive lichen planus, allergic dermatitis, auto-immune bullous dermatoses, drug-reactions. Chronic balanitis, or recurrent inflammatory balanitis, lichen sclerosus may lead to the emergence of intraepithelial neoplasias (PIN) or invasive carcinomas. The management of genital lesions needs in fact: the observation or oral mucous membrances and of the skin it-self with a special attention to very peculiar body sites for the presence of typical skin disease lesions; a surgical biopsy for microscopic observation; in some cases immunopathology with direct and indirect immunofluorescence for the diagnosis of auto-immune disorders.
\end{abstract}

Key-words: balanitis, carcinoma, dermatology, lichen, penis 